\title{
NOTICE
}

\section{Psychonomic Society Publications Office Address Change}

The Psychonomic Society Publications Office is pleased to announce its relocation as of June 15, 1987. Please address all future correspondence to this new address:

Psychonomic Society Publications

1710 Fortview Road

Austin, TX 78704 